\title{
On Bounds for Moments of Unimodal Distributions
}

\author{
R. Sharma ${ }^{1, a}$, R. Bhandari ${ }^{a}$ \\ ${ }^{a}$ Department of Mathematics \& Statistics, Himachal Pradesh University, India
}

\begin{abstract}
We provide a simple basic method to find bounds for higher order moments of unimodal distributions in terms of lower order moments when the random variable takes value in a given finite real interval. The bounds for moments in terms of the geometric mean of the distribution are also derived. Both continuous and discrete cases are considered. The bounds for the ratio and difference of moments are obtained. The special cases provide refinements of several well-known inequalities, such as Kantorovich inequality and Krasnosel'skii and Krein inequality.

Keywords: Discrete distributions, harmonic mean, Kantorovich inequality, moments, unimodal distributions, variance.
\end{abstract}

\section{Introduction}

The $r^{\text {th }}$ order moment $\mu_{r}^{\prime}$ of a random variable $X$ in $[a, b]$ for the continuous and discrete cases respectively are defined as

$$
\mu_{r}^{\prime}=\int_{a}^{b} x^{r} \phi(x) d x \quad \text { or } \quad \mu_{r}^{\prime}=\sum_{i=1}^{n} p_{i} x_{i}^{r},
$$

where $\phi(x)$ and $p_{i}$ are corresponding probability densities and probability functions such that

$$
\int_{a}^{b} \phi(x) d x=1 \quad \text { or } \quad \sum_{i=1}^{n} p_{i}=1 .
$$

A distribution is unimodal with mode $M$ if $\phi(x)$ is non-decreasing in $[a, M)$ and non-increasing in $(M, b]$.The inequalities involving moments of arbitrary distributions have been studied extensively in literature. The related bounds for the unimodal distributions are mainly discussed for the variance of the distribution. Gray and Odell (1967) prove that for a symmetrical unimodal distribution

$$
\sigma^{2} \leq \frac{(b-a)^{2}}{12}
$$

where $\sigma^{2}$ is the variance of the distribution, $\sigma^{2}=\mu_{2}^{\prime}-\mu_{1}^{\prime 2}$. Jacobson (1969) shows that the bound (1.3) remains valid for distribution with mode at $x=(a+b) / 2$. Jacobson (1969) also shows that for a unimodal distribution

$$
\sigma^{2} \leq \frac{(b-a)^{2}}{9}
$$

The support of the UGC-SAP is acknowledged.

${ }^{1}$ Corresponding author: Department of Mathematics \& Statistics, Himachal Pradesh University, Shimla-5, 171005, India.

E-mail: rajesh_hpu_math@yahoo.co.in 
The derivations given by Jacobson (1969) were lengthy and tedious; subsequently, alternative proofs of inequality (1.4) were considered by Seaman et al. (1987) and Dharmadhikari and Joag-Dev (1989). For the further related developments and refinements of these inequalities, see Dharmadhikari and Joag-Dev (1989) and Sharma and Bhandari (2013).

A discrete distribution $\left\{p_{1}, p_{2}, \ldots, p_{n}\right\}$ with support $\left\{x_{1}, x_{2}, \ldots, x_{n}\right\}$ such that $x_{1} \leq x_{2} \leq \cdots \leq x_{n}$ is unimodal about $x=x_{k}$ if $p_{1} \leq p_{2} \leq \cdots \leq p_{k} \geq p_{k+1} \geq \cdots \geq p_{n}$, see Keilson and Gerber (1971) and Medgyessy (1972). Abouammoh and Mashhour (1994) have obtained the upper bound for the variance of discrete unimodal distribution with support $\{0,1,2, \ldots, n\}$. We pursue this topic further and consider the bounds for moments on arbitrary support $\left\{x_{1}, x_{2}, \ldots, x_{n}\right\}$.

Sharma and Bhandari (2013) have recently given alternative and elementary proofs of the variance bounds for continuous unimodal distributions and derived the analogous bounds for discrete distributions with arbitrary support $\left\{x_{1}, x_{2}, \ldots, x_{n}\right\}$. In a similar spirit, we obtain here bounds for the higher order moments and inequalities involving the ratio and difference of moments of unimodal distributions.

Our main results give the bounds for the $r^{\text {th }}$ order moment $\mu_{r}^{\prime}$ in terms of the sth order moment $\mu_{s}^{\prime}$ for both continuous and discrete unimodal distributions (Theorem 1-2). Likewise, we give the bounds for $\mu_{r}^{\prime}$ in terms of the geometric mean of the distributions (Theorem 3-4). As a consequence we get several inequalities involving difference and ratio of moments (Corollary 1-4). The special cases of interest are discussed and we get refinements and alternative proofs of the inequalities given by Krasnosel'skii and Krein (1952), Kantorovich (1948) and Shisha and Mond (1967).

\section{Main Results}

Lemma 1. (Sharma et al., 2004) If $r$ is a positive real number and $s$ is a non-zero real number with $r>s$ then for $0<a \leq x \leq b$, we have

$$
x^{r} \leq \frac{b^{r}-a^{r}}{b^{s}-a^{s}} x^{s}-\frac{a^{s} b^{r}-a^{r} b^{s}}{b^{s}-a^{s}}, \quad a \neq b,
$$

and for $x$ lying outside $(a, b)$ the inequality (2.1) reverses. If $r$ is a negative real number with $r>s$, the inequality (2.1) holds for $x$ lying outside $(a, b)$ and reverses for $a \leq x \leq b$.

Theorem 1. Let $X$ be a continuous random variable having unimodal distribution about $M$. Let $0<a \leq X \leq b, a \neq b$. If $r$ is a positive real number and $s$ is a non-zero real number such that $r>s$, then

$$
\mu_{r}^{\prime} \leq \frac{m_{r(M, b)}^{\prime}-m_{r(a, M)}^{\prime}}{m_{s(M, b)}^{\prime}-m_{s(a, M)}^{\prime}} \mu_{s}^{\prime}-\frac{m_{s(a, M)}^{\prime} m_{r(M, b)}^{\prime}-m_{r(a, M)}^{\prime} m_{s(M, b)}^{\prime}}{m_{s(M, b)}^{\prime}-m_{s(a, M)}^{\prime}}
$$

where

$$
m_{r(c, d)}^{\prime}=\frac{1}{d-c} \int_{c}^{d} x^{r} d x .
$$

If $r$ and $s$ are negative real numbers with $r>s$, the inequality (2.2) reverses.

Proof: Let

$$
I=\int_{a}^{b}\left(x^{r}-A x^{s}+B\right) \phi(x) d x
$$


where $A=\left(\gamma^{r}-\beta^{r}\right) /\left(\gamma^{s}-\beta^{s}\right)$ and $B=\left(\beta^{s} \gamma^{r}-\gamma^{s} \beta^{r}\right) /\left(\gamma^{s}-\beta^{s}\right)$. For $a \leq \beta \leq M \leq \gamma \leq b$, we have

$$
\begin{aligned}
I= & \int_{a}^{\beta}\left(x^{r}-A x^{s}+B\right) \phi(x) d x-\int_{\beta}^{M}\left(-x^{r}+A x^{s}-B\right) \phi(x) d x \\
& -\int_{M}^{\gamma}\left(-x^{r}+A x^{s}-B\right) \phi(x) d x+\int_{\gamma}^{b}\left(x^{r}-A x^{s}+B\right) \phi(x) d x .
\end{aligned}
$$

It follows from Lemma 1 that each integrand in (2.3) is non-negative. Further, the unimodality of $\phi(x)$ at $x=M$ implies that $\phi(x) \leq \phi(\beta)$ in $[a, \beta], \phi(x) \geq \phi(\beta)$ in $[\beta, M], \phi(x) \geq \phi(\gamma)$ in $[M, \gamma]$ and $\phi(x) \leq \phi(\gamma)$ in $[\gamma, b]$. Therefore

$$
I \leq \phi(\beta) \int_{a}^{M}\left(x^{r}-A x^{s}+B\right) d x+\phi(\gamma) \int_{M}^{b}\left(x^{r}-A x^{s}+B\right) d x .
$$

We choose $\beta$ and $\gamma$ such that

$$
\int_{a}^{M}\left(x^{r}-A x^{s}+B\right) d x=0
$$

and

$$
\int_{M}^{b}\left(x^{r}-A x^{s}+B\right) d x=0
$$

From (2.5)

$$
m_{r(a, M)}^{\prime}-m_{s(a, M)}^{\prime} A+B=0
$$

for $M>a$. For $M<b$, we have

$$
m_{r(M, b)}^{\prime}-m_{s(M, b)}^{\prime} A+B=0 .
$$

The solution of the system of equations (2.7) and (2.8) gives

$$
A=\frac{m_{r(M, b)}^{\prime}-m_{r(a, M)}^{\prime}}{m_{s(M, b)}^{\prime}-m_{s(a, M)}^{\prime}}
$$

and

$$
B=\frac{m_{s(a, M)}^{\prime} m_{r(M, b)}^{\prime}-m_{r(a, M)}^{\prime} m_{s(M, b)}^{\prime}}{m_{s(M, b)}^{\prime}-m_{s(a, M)}^{\prime}}
$$

On the other hand if (2.5) and (2.6) hold, the inequality (2.4) gives

$$
\mu_{r}^{\prime} \leq A \mu_{s}^{\prime}-B .
$$

Combine (2.9), (2.10) and (2.11) we immediately get (2.2). Likewise, we can discuss the case when $r$ and $s$ are negative real numbers, $r>s$. 
For a non-increasing distribution $(M=a)$, the upper bound for $\mu_{r}^{\prime}$ follows as a limiting case of (2.2),

$$
\mu_{r}^{\prime} \leq \frac{m_{r(a, b)}^{\prime}-a^{r}}{m_{s(a, b)}^{\prime}-a^{s}} \mu_{s}^{\prime}-\frac{a^{s} m_{r(a, b)}^{\prime}-a^{r} m_{s(a, b)}^{\prime}}{m_{s(a, b)}^{\prime}-a^{s}} .
$$

Likewise, for $M=b$,

$$
\mu_{r}^{\prime} \leq \frac{m_{r(a, b)}^{\prime}-b^{r}}{m_{s(a, b)}^{\prime}-b^{s}} \mu_{s}^{\prime}-\frac{b^{s} m_{r(a, b)}^{\prime}-b^{r} m_{s(a, b)}^{\prime}}{m_{s(a, b)}^{\prime}-b^{s}} .
$$

Theorem 2. Let $p_{n}$ be a discrete unimodal distribution about $x_{k}$ with support $\left\{x_{1}, x_{2}, \ldots, x_{n}\right\}, n \geq 3$ such that $0<x_{1} \leq x_{2} \leq \cdots \leq x_{n}$. If $r$ is a positive real number and $s$ is a non-zero real number such that $r>s$, then

$$
\mu_{r}^{\prime} \leq \min _{i=1,2}\left(A_{i} \mu_{s}^{\prime}-B_{i}\right)
$$

where

$$
\begin{array}{ll}
A_{1}=\frac{m_{r(1, n)}^{\prime}-m_{r(1, k)}^{\prime}}{m_{s(1, n)}^{\prime}-m_{s(1, k)}^{\prime}}, & B_{1}=\frac{m_{s(1, k)}^{\prime} m_{r(1, n)}^{\prime}-m_{s(1, n)}^{\prime} m_{r(1, k)}^{\prime}}{m_{s(1, n)}^{\prime}-m_{s(1, k)}^{\prime}}, \quad k<n, \\
A_{2}=\frac{m_{r(1, n)}^{\prime}-m_{r(k, n)}^{\prime}}{m_{s(1, n)}^{\prime}-m_{s(k, n)}^{\prime}}, & B_{2}=\frac{m_{s(k, n)}^{\prime} m_{r(1, n)}^{\prime}-m_{s(1, n)}^{\prime} m_{r(k, n)}^{\prime}}{m_{s(1, n)}^{\prime}-m_{s(k, n)}^{\prime}}, \quad k>1
\end{array}
$$

and

$$
m_{r(l, t)}^{\prime}=\frac{1}{t-l+1} \sum_{i=l}^{t} x_{i}^{r}
$$

If $r$ and $s$ are negative real numbers with $r>s$, the reverse inequality of (2.12) holds with max instead of min.

Proof: For $0<x_{1} \leq x_{\beta} \leq x_{k} \leq x_{\gamma} \leq x_{n}$, we write

$$
\begin{aligned}
\sum_{i=1}^{n} p_{i}\left(x_{i}^{r}-\frac{v^{r}-u^{r}}{v^{s}-u^{s}} x_{i}^{s}+\frac{u^{s} v^{r}-u^{r} v^{s}}{v^{s}-u^{s}}\right)= & \sum_{i=1}^{n} p_{i}\left(x_{i}^{r}-U x_{i}^{s}+V\right) \\
= & \sum_{i=1}^{\beta} p_{i}\left(x_{i}^{r}-U x_{i}^{s}+V\right)-\sum_{i=\beta+1}^{k} p_{i}\left(-x_{i}^{r}+U x_{i}^{s}-V\right) \\
& -\sum_{i=k+1}^{\gamma} p_{i}\left(-x_{i}^{r}+U x_{i}^{s}-V\right)+\sum_{i=\gamma+1}^{n} p_{i}\left(x_{i}^{r}-U x_{i}^{s}+V\right),
\end{aligned}
$$

where $U=\left(v^{r}-u^{r}\right) /\left(v^{s}-u^{s}\right)$ and $V=\left(u^{s} v^{r}-u^{r} v^{s}\right) /\left(v^{s}-u^{s}\right)$. It follows from Lemma 1 that for $x_{\beta} \leq u \leq x_{\beta+1}$ and $x_{\gamma} \leq v \leq x_{\gamma+1}$ each summand in (2.15) is non-negative. Further, the distribution is 
unimodal at $x_{k}$ therefore $p_{i} \leq p_{\beta}$ for $i=1,2, \ldots, \beta$ and $p_{i} \geq p_{\beta}$ for $i=\beta+1, \beta+2, \ldots, k$. Likewise, $p_{i} \geq p_{\gamma}$ for $i=k+1, k+2, \ldots, \gamma$ and $p_{i} \leq p_{\gamma}$ for $i=\gamma+1, \gamma+2, \ldots, n$. Therefore

$$
\sum_{i=1}^{n} p_{i}\left(x_{i}^{r}-U x_{i}^{s}+V\right) \leq p_{\beta} \sum_{i=1}^{k}\left(x_{i}^{r}-U x_{i}^{s}+V\right)+p_{\gamma} \sum_{i=k+1}^{n}\left(x_{i}^{r}-U x_{i}^{s}+V\right) .
$$

We choose $\beta$ and $\gamma$ such that

$$
\sum_{i=1}^{k}\left(x_{i}^{r}-U x_{i}^{s}+V\right)=0
$$

and

$$
\sum_{i=k+1}^{n}\left(x_{i}^{r}-U x_{i}^{s}+V\right)=0 .
$$

From (2.17) and (2.18) we respectively get that

$$
\sum_{i=1}^{k} x_{i}^{r}-U \sum_{i=1}^{k} x_{i}^{s}+k V=0
$$

and

$$
\sum_{i=k+1}^{n} x_{i}^{r}-U \sum_{i=k+1}^{n} x_{i}^{s}+(n-k) V=0
$$

So,

$$
U=\frac{(n-k) \sum_{i=1}^{k} x_{i}^{r}-k \sum_{i=k+1}^{n} x_{i}^{r}}{(n-k) \sum_{i=1}^{k} x_{i}^{s}-k \sum_{i=k+1}^{n} x_{i}^{s}}
$$

and

$$
V=\frac{\sum_{i=1}^{k} x_{i}^{s} \sum_{i=k+1}^{n} x_{i}^{r}-\sum_{i=k+1}^{n} x_{i}^{s} \sum_{i=1}^{k} x_{i}^{r}}{k \sum_{i=k+1}^{n} x_{i}^{s}-(n-k) \sum_{i=1}^{k} x_{i}^{s}} .
$$

A simple calculation shows that $U=A_{1}$ and $V=B_{1}, A_{1}$ and $B_{1}$ are given in (2.13). On the other hand if (2.17) and (2.18) hold, the inequality (2.16) gives

$$
\mu_{r}^{\prime} \leq A_{1} \mu_{s}^{\prime}-B_{1} .
$$

The inequality $\mu_{r}^{\prime} \leq A_{2} \mu_{s}^{\prime}-B_{2}$ follows on using similar arguments and the fact that (2.15) can be written as,

$$
\begin{aligned}
\sum_{i=1}^{n} p_{i}\left(x_{i}^{r}-U x_{i}^{s}+V\right)= & \sum_{i=1}^{\beta} p_{i}\left(x_{i}^{r}-U x_{i}^{s}+V\right)-\sum_{i=\beta+1}^{k-1} p_{i}\left(-x_{i}^{r}+U x_{i}^{s}-V\right) \\
& -\sum_{i=k}^{\gamma} p_{i}\left(-x_{i}^{r}+U x_{i}^{s}-V\right)+\sum_{i=\gamma+1}^{n} p_{i}\left(x_{i}^{r}-U x_{i}^{s}+V\right) .
\end{aligned}
$$


Likewise, it follows on using similar arguments and Lemma 1 that (2.12) reverses its order when $r$ and $s$ are negative real numbers with $r>s$.

Note that in the preceding theorems we have assumed that $s \neq 0$. This particular case $(s=0)$ gives the bounds for $r^{\text {th }}$ order moment $\mu_{r}^{\prime}$ in terms of the geometric mean $(G)$ of the unimodal distribution. These bounds can be derived on using arguments similar to those used in Theorem 1-2. We mention them here without proofs in the following theorems.

Theorem 3. Let $X$ be a continuous random variable having unimodal distribution about $M$. Let $0<a \leq X \leq b, a \neq b$. Then for every real number $r$,

$$
\mu_{r}^{\prime} \leq \frac{m_{r(M, b)}^{\prime}-m_{r(a, M)}^{\prime}}{\log m_{0(M, b)}-\log m_{0(a, M)}} \log G-\frac{m_{r(M, b)}^{\prime} \log m_{0(a, M)}-m_{r(a, M)}^{\prime} \log m_{0(M, b)}}{\log m_{0(M, b)}-\log m_{0(a, M)}},
$$

where

$$
\log m_{0(c, d)}=\frac{1}{d-c} \int_{c}^{d} \log x d x .
$$

Theorem 4. Let $p_{n}$ be a discrete unimodal distribution about $x_{k}$ with support $\left\{x_{1}, x_{2}, \ldots, x_{n}\right\}, n \geq 3$ such that $0<x_{1} \leq x_{2} \leq \cdots \leq x_{n}$. Then for every real number $r$,

$$
\mu_{r}^{\prime} \leq \min _{i=1,2}\left(E_{i} \log G-F_{i}\right),
$$

where

$$
\begin{array}{lll}
E_{1}=\frac{m_{r(1, n)}^{\prime}-m_{r(1, k)}^{\prime}}{\log m_{0(1, n)}-\log m_{0(1, k)}}, & F_{1}=\frac{m_{r(1, n)}^{\prime} \log m_{0(1, k)}-m_{r(1, k)}^{\prime} \log m_{0(1, n)}}{\log m_{0(1, n)}-\log m_{0(1, k)}}, & k<n, \\
E_{2}=\frac{m_{r(1, n)}^{\prime}-m_{r(k, n)}^{\prime}}{\log m_{0(1, n)}-\log m_{0(k, n)}}, & F_{2}=\frac{m_{r(1, n)}^{\prime} \log m_{0(k, n)}-m_{r(k, n)}^{\prime} \log m_{0(1, n)}}{\log m_{0(1, n)}-\log m_{0(k, n)}}, & k>1
\end{array}
$$

and

$$
\log m_{0(l, t)}=\frac{1}{t-l+1} \sum_{i=l}^{t} \log x_{i} .
$$

We now show that the bounds for the difference and ratio of moments can be derived easily from inequalities obtained in the above theorems.

Corollary 1. Under the conditions of Theorem 1

$$
\mu_{r}^{\prime}-\left(\mu_{s}^{\prime}\right)^{\frac{r}{s}} \leq A\left(\frac{s}{r} A\right)^{\frac{s}{r-s}}-\left(\frac{s}{r} A\right)^{\frac{r}{r-s}}-B
$$

where $r$ is a positive real number and $s$ is a non-zero real number such that $r>s$. If $r$ and $s$ are negative real numbers with $r>s$, the inequality (2.23) reverses.

Proof: The inequality (2.23) follows easily from Theorem 1. From (2.2),

$$
\mu_{r}^{\prime}-\left(\mu_{s}^{\prime}\right)^{\frac{r}{s}} \leq A \mu_{s}^{\prime}-\left(\mu_{s}^{\prime}\right)^{\frac{r}{s}}-B
$$


and the right hand side expression in (2.24) achieves its maximum at $\mu_{s}^{\prime}=((s / r) A)^{s /(r-s)}$.

Corollary 2. Under the conditions of Theorem 2,

$$
\mu_{r}^{\prime}-\left(\mu_{s}^{\prime}\right)^{\frac{r}{s}} \leq \min _{i=1,2}\left\{A_{i}\left(\frac{s}{r} A_{i}\right)^{\frac{s}{r-s}}-\left(\frac{s}{r} A_{i}\right)^{\frac{r}{r-s}}-B_{i}\right\},
$$

where $r$ is a positive real number and $s$ is a non-zero real number such that $r>s$. For the case when $r$ and $s$ are negative real numbers with $r>s$ the reverse inequality of (2.25) holds with max instead of $\min$.

Proof: Use Theorem 2 and arguments similar to those used in the proof of Corollary 1.

Likewise, bounds for $\mu_{r}^{\prime}-G^{r}$ follow from inequalities (2.19) and (2.20). Under the conditions of Theorem 3, we have, for $r \neq 0$,

$$
\mu_{r}^{\prime}-G^{r} \leq\left(\log \frac{L_{1}}{r}-1\right) \frac{L_{1}}{r}-L_{2}
$$

where

$$
L_{1}=\frac{m_{r(M, b)}^{\prime}-m_{r(a, M)}^{\prime}}{\log m_{0(M, b)}-\log m_{0(a, M)}}, \quad L_{2}=\frac{m_{r(M, b)}^{\prime} \log m_{0(a, M)}-m_{r(a, M)}^{\prime} \log m_{0(M, b)}}{\log m_{0(M, b)}-\log m_{0(a, M)}} .
$$

Under the conditions of Theorem 4, we have

$$
\mu_{r}^{\prime}-G^{r} \leq \min _{i=1,2}\left\{\left(\log \frac{E_{i}}{r}-1\right) \frac{E_{i}}{r}-F_{i}\right\}
$$

where $E_{i}$ and $F_{i}(i=1,2)$ are given in (2.21) and (2.22).

Corollary 3. Under the conditions of Theorem 1

$$
\frac{\mu_{r}^{\prime}}{\left(\mu_{s}^{\prime}\right)^{\frac{r}{s}}} \leq A\left(\frac{r}{r-s} \frac{B}{A}\right)^{1-\frac{r}{s}}-B\left(\frac{r}{r-s} \frac{B}{A}\right)^{-\frac{r}{s}}
$$

where $r$ is a positive real number and $s$ is a non-zero real number such that $r>s$. If $r$ and $s$ are negative real numbers with $r>s$, the inequality (2.29) reverses.

Proof: The inequality (2.29) follows easily from Theorem 1. From (2.2),

$$
\frac{\mu_{r}^{\prime}}{\left(\mu_{s}^{\prime}\right)^{\frac{r}{s}}} \leq A\left(\mu_{s}^{\prime}\right)^{1-\frac{r}{s}}-B\left(\mu_{s}^{\prime}\right)^{-\frac{r}{s}}
$$

and the right hand side expression in (2.30) achieves its maximum at $\mu_{s}^{\prime}=r /(r-s)(B / A)$.

Corollary 4. Under the conditions of Theorem 2,

$$
\frac{\mu_{r}^{\prime}}{\left(\mu_{s}^{\prime}\right)^{\frac{r}{s}}} \leq \min _{i=1,2}\left\{A_{i}\left(\frac{r}{r-s} \frac{B_{i}}{A_{i}}\right)^{1-\frac{r}{s}}-B_{i}\left(\frac{r}{r-s} \frac{B_{i}}{A_{i}}\right)^{-\frac{r}{s}}\right\}
$$


where $r$ is a positive real number and $s$ is a non-zero real number such that $r>s$. For the case when $r$ and $s$ are negative real numbers with $r>s$ the reverse inequality of (2.31) holds with max instead of $\min$.

Proof: Use Theorem 2 and arguments similar to those used in the proof of Corollary 3.

Likewise, bounds for $\mu_{r}^{\prime} / G^{r}$ follow from inequalities (2.19) and (2.20). Under the conditions of Theorem 3, for $r \neq 0$,

$$
\frac{\mu_{r}^{\prime}}{G^{r}} \leq \frac{L_{1}}{r} e^{-\frac{L_{1}+r L_{2}}{L_{1}}},
$$

where $L_{1}$ and $L_{2}$ are given in (2.27). Under the conditions of Theorem 4 , for $r \neq 0$,

$$
\frac{\mu_{r}^{\prime}}{G^{r}} \leq \min _{i=1,2} \frac{E_{i}}{r} e^{-\frac{E_{i}+r F_{i}}{E_{i}}},
$$

where $E_{i}$ and $F_{i}(i=1,2)$ are given in (2.21) and (2.22).

\section{Special Cases: Refinements of Related Inequalities}

The bounds for the variance can be studied as special cases of the more general results obtained here for the $r^{\text {th }}$ central moment. For example, it follows from the inequality (2.2) that for $r=2$ and $s=1$, we have

$$
3 \sigma^{2} \leq\left(b-\mu_{1}^{\prime}\right)\left(\mu_{1}^{\prime}-a\right)-\left(M-\mu_{1}^{\prime}\right)\left(a+b-2 \mu_{1}^{\prime}\right)
$$

where $\sigma^{2}=\mu_{2}^{\prime}-\mu_{1}^{\prime 2}$.

The special cases of the above results also provide refinements of the several well-known inequalities that involve moments of both continuous and discrete distributions. We demonstrate some of these cases here.

For a discrete or continuous random variable $X$ such that $0<a \leq X \leq b$, the Krasnosel'skii and Krein (1952) inequality says that

$$
\frac{\mu_{2}^{\prime}}{\mu_{1}^{\prime 2}} \leq \frac{(a+b)^{2}}{4 a b} .
$$

The inequality (2.29) provides a refinement of the inequality (3.1), $r=2$ and $s=1$,

$$
\frac{\mu_{2}^{\prime}}{\mu_{1}^{\prime 2}} \leq \frac{(a+b+M)^{2}}{3(a b+(a+b) M)} .
$$

The important special cases of unimodal distribution namely $\phi(x)$ is non-increasing or non-decreasing in $[a, b]$ and $\phi(x)$ is unimodal at $(a+b) / 2$ have been studied explicitly in literature, see Gray and Odell (1967), Dharmadhikari and Joag-Dev (1989) and Sharma and Bhandari (2013). Here, if $\phi(x)$ is non-increasing in $[a, b]$, then $M=a$ and (3.2) gives

$$
\frac{\mu_{2}^{\prime}}{\mu_{1}^{\prime 2}} \leq \frac{(a+b)^{2}}{4 a b}-\frac{(b-a)^{2}(3 a+2 b)}{12 a b(a+2 b)} .
$$


The inequality (3.3) clearly affects an improvement on (3.1). Similarly, if $\phi(x)$ is non-decreasing in $[a, b]$,

$$
\frac{\mu_{2}^{\prime}}{\mu_{1}^{\prime 2}} \leq \frac{(a+b)^{2}}{4 a b}-\frac{(b-a)^{2}(3 b+2 a)}{12 a b(b+2 a)} .
$$

Also, if distribution is unimodal at $M=(a+b) / 2$,

$$
\frac{\mu_{2}^{\prime}}{\mu_{1}^{\prime 2}} \leq \frac{(a+b)^{2}}{4 a b}-\frac{\left(b^{2}-a^{2}\right)^{2}}{4 a b\left((a+b)^{2}+2 a b\right)} .
$$

Likewise, we can discuss the refinement of the inequality (3.1) for discrete distributions. For example, if distribution is unimodal at $x=x_{k}$ and $x_{i} \geq 0, i=1,2, \ldots, n$, the inequality (2.31) gives, $r=2$ and $s=1$,

$$
\frac{\mu_{2}^{\prime}}{\mu_{1}^{\prime 2}} \leq \min \left\{\frac{\left(a_{1}+b_{1}\right)^{2}}{4 a_{1} b_{1}}, \frac{\left(a_{2}+b_{2}\right)^{2}}{4 a_{2} b_{2}}\right\}
$$

where

$$
a_{i}=\frac{A_{i}-\sqrt{A_{i}^{2}-4 B_{i}}}{2} \text { and } b_{i}=\frac{A_{i}+\sqrt{A_{i}^{2}-4 B_{i}}}{2},
$$

$A_{i}$ and $B_{i}(i=1,2)$ are given in (2.13) and (2.14).

The Kantorovich (1948) inequality says that if a random variable, discrete or continuous, takes values in the interval $[a, b], a>0$, then

$$
\frac{\mu_{1}^{\prime}}{H} \leq \frac{(a+b)^{2}}{4 a b},
$$

where $H=\left(\mu_{-1}^{\prime}\right)^{-1}$ is the Harmonic mean. A refinement of inequality (3.5) for continuous unimodal distributions follows from Corollary $3, r=1, s=-1$,

$$
\frac{\mu_{1}^{\prime}}{H} \leq \frac{\left(a_{3}+b_{3}\right)^{2}}{4 a_{3} b_{3}} \text {, }
$$

where

$$
a_{3}=\frac{-B-\sqrt{B^{2}+4 A}}{2} \text { and } \quad b_{3}=\frac{-B+\sqrt{B^{2}+4 A}}{2},
$$

$A$ and $B$ are respectively given in (2.9) and (2.10).

A refinement of inequality (3.5) for discrete distributions, follows from Corollary 4, $r=1, s=-1$,

$$
\frac{\mu_{1}^{\prime}}{H} \leq \min \left\{\frac{\left(a_{4}+b_{4}\right)^{2}}{4 a_{4} b_{4}}, \frac{\left(a_{5}+b_{5}\right)^{2}}{4 a_{5} b_{5}}\right\},
$$

where

$$
a_{i+3}=\frac{-B_{i}-\sqrt{B_{i}^{2}+4 A_{i}}}{2} \quad \text { and } \quad b_{i+3}=\frac{-B_{i}+\sqrt{B_{i}^{2}+4 A_{i}}}{2}, \quad i=1,2
$$


$A_{i}$ and $B_{i}(i=1,2)$ are given in (2.13) and (2.14).

For a discrete or continuous random variable $X$ such that $0<a \leq X \leq b$, Shisha and Mond (1967) proved that

$$
\mu_{1}^{\prime}-H \leq(\sqrt{b}-\sqrt{a})^{2} .
$$

For continuous unimodal distributions, Corollary 1 gives a refinement,

$$
\mu_{1}^{\prime}-H \leq\left(\sqrt{b_{3}}-\sqrt{a_{3}}\right)^{2},
$$

where $a_{3}$ and $b_{3}$ are given in (3.6). For discrete case, Corollary 2 gives

$$
\mu_{1}^{\prime}-H \leq \min \left\{\left(\sqrt{b_{4}}-\sqrt{a_{4}}\right)^{2},\left(\sqrt{b_{5}}-\sqrt{a_{5}}\right)^{2}\right\},
$$

where $a_{4}, b_{4}$ and $a_{5}, b_{5}$ are given in (3.7).

Shisha and Mond (1967) have also proved that if a discrete or continuous random variable $X$ is such that $0<a \leq X \leq b$, then

$$
\sqrt{\mu_{2}^{\prime}}-\mu_{1}^{\prime} \leq \frac{(b-a)^{2}}{4(a+b)} .
$$

We prove a refinement of the inequality (3.8) for continuous unimodal distributions in the following theorem.

Theorem 5. Let $X$ be a continuous random variable having unimodal distribution about M. For $0<a \leq X \leq b$,

$$
\sqrt{\mu_{2}^{\prime}}-\mu_{1}^{\prime} \leq \frac{\left(b_{6}-a_{6}\right)^{2}}{4\left(a_{6}+b_{6}\right)}
$$

where

$$
a_{6}=\frac{A-\sqrt{A^{2}-4 B}}{2} \quad \text { and } \quad b_{6}=\frac{A+\sqrt{A^{2}-4 B}}{2},
$$

$A$ and $B$ are respectively given in (2.9) and (2.10), $r=2, s=1$.

Proof: From (2.2),

$$
\sqrt{\mu_{2}^{\prime}}-\mu_{1}^{\prime} \leq \sqrt{\left(a_{6}+b_{6}\right) \mu_{1}^{\prime}-a_{6} b_{6}}-\mu_{1}^{\prime} .
$$

The function

$$
f(x)=\sqrt{(l+m) x-l m}-x
$$

with derivative

$$
f^{\prime}(x)=\frac{(l+m)-2 \sqrt{(l+m) x-l m}}{2 \sqrt{(l+m) x-l m}}
$$


vanishes at

$$
x=\frac{(l+m)^{2}+4 l m}{4(l+m)}
$$

where $f(x)$ achieves its maximum value. It follows that

$$
f(x) \leq \frac{(m-l)^{2}}{4(l+m)} .
$$

Combine (3.10), (3.11) and (3.12); the inequality (3.9) follows immediately.

Likewise, a refinement of inequality (3.8) for discrete distributions follows from Theorem 2,

$$
\sqrt{\mu_{2}^{\prime}}-\mu_{1}^{\prime} \leq \min \left\{\frac{\left(b_{1}-a_{1}\right)^{2}}{4\left(a_{1}+b_{1}\right)}, \frac{\left(b_{2}-a_{2}\right)^{2}}{4\left(a_{2}+b_{2}\right)}\right\},
$$

where $a_{1}, b_{1}$ and $a_{2}, b_{2}$ are given in (3.4).

\section{Summary and Discussion}

Sharma and Bhandari (2013) have recently given alternative and simple proofs of the bounds on the variance of unimodal distributions. It is natural to consider the generalizations of these inequalities for higher order moments. Enhancing the technique of Sharma and Bhandari (2013) we have been able to find the bounds for the moments of unimodal distributions. One advantage of the method is that it is uniformly applicable to both continuous and discrete unimodal distributions. Moreover, all the bounds are tight as the corresponding inequalities becomes equalities for uniform distributions. For example, a simple calculation shows that for uniform distribution

$$
\frac{m_{r(M, b)}^{\prime}-m_{r(a, M)}^{\prime}}{m_{s(M, b)}^{\prime}-m_{s(a, M)}^{\prime}} \mu_{s}^{\prime}-\frac{m_{s(a, M)}^{\prime} m_{r(M, b)}^{\prime}-m_{r(a, M)}^{\prime} m_{s(M, b)}^{\prime}}{m_{s(M, b)}^{\prime}-m_{s(a, M)}^{\prime}}=\frac{b^{r+1}-a^{r+1}}{(r+1)(b-a)} .
$$

So, the inequality (2.2) become equality. Hence, (2.2) is best. Further, in some applications it is of interest to know the limits of moments, when the exact form of a distribution is not derivable, although the distribution is known to be unimodal. The above inequalities can be used to find the estimates for the variance and moments of unimodal distribution. We explain this by means of following examples.

Example 1. For a $\beta$-distribution

$$
\phi(x)=\frac{\lceil(\alpha+\beta)}{\lceil(\alpha)\lceil(\beta)} x^{\alpha-1}(1-x)^{\beta-1}, \quad 0<x<1 .
$$

The distribution is unimodal with mode $(\alpha-1) /(\alpha+\beta-2)$, where $\alpha, \beta>1$. On using above inequalities, we can write several inequalities for the moments, ratio of moments and difference of moments, without using the probability density function given in (4.1). For example, for $\alpha=\beta=2$, it follows from (2.2) that $\mu_{r}^{\prime} \leq 1 /(r+1)$ for every real number $r \geq 1$. Note that the $r^{\text {th }}$ moment of $\beta$-distribution is $\mu_{r}^{\prime}=\prod_{i=0}^{r-1}(2+i) /(4+i)$, and the inequality $\mu_{r}^{\prime} \leq 1 /(r+1)$ follows easily for $r=1,2, \ldots$.

Example 2. For a Binomial distribution

$$
p_{i}= \begin{cases}{ }^{n} C_{i} p^{i}(1-p)^{n-i}, & i=0,1,2, \ldots, n, \\ 0, & \text { otherwise. }\end{cases}
$$


The distribution is unimodal when $(n+1) p$ is not an integer. If $p>1 /(n+2)$, the distribution is non-increasing. Therefore, for all $p>1 /(n+2)$, it follows from the inequality (2.12) that $\mu_{r}^{\prime} \leq$ $2 p /(n+1) \sum_{i=0}^{n} i^{r}$. Similarly, we can discuss various cases and inequalities for the moments of this distribution.

Example 3. The distribution

$$
p_{i}= \begin{cases}\frac{4-i}{6}, & \text { for } i=1,2,3, \\ 0, & \text { elsewhere }\end{cases}
$$

with support $x_{i}=\{\ldots,-4,3,6, \ldots\}$ is unimodal at $x_{1}=-4$. The mean of the distribution is $\mu_{1}^{\prime}=0$. From the Popoviciu (1935) inequality $(\sigma \leq(b-a) / 2)$, we have $\sigma \leq 5$ and from (2.12) we have $\sigma \leq \sqrt{324 / 17}=4.3656, r=2$. The inequality (2.12) therefore gives better estimate. Also, from (2.12), $\mu_{r}^{\prime} \leq\left\{4\left(3^{r}+6^{r}\right)+9(-4)^{r}\right\} / 17$.

\section{Acknowledgements}

The authors thank the I.S.I.-Delhi for a visit in January 2012 when this work was begun.

\section{References}

Abouammoh, A. M. and Mashhour, A. F. (1994). Variance upper bounds and convolutions of $\alpha$ unimodal distributions, Statistics \& Probability Letters, 21, 281-289.

Dharmadhikari, S. W. and Joag-Dev, K. (1989). Upper bounds for the variances of certain random variables, Communications in Statistics - Theory and Methods, 18, 3235-3247.

Gray, H. L. and Odell, P. L. (1967). On least favorable density functions, SIAM Review, 9, 715-720.

Jacobson, H. I. (1969). The maximum variance of restricted unimodal distributions, Annals of Mathematical Statistics, 40, 1746-1752.

Kantorovich, L. V. (1948). Functional analysis and applied mathematics [in Russian], Uspekki Matematicheskikh Nauk, 3, 89-185.

Keilson, J. and Gerber, H. (1971). Some results for discrete unimodality, Journal of the American Statistical Association, 66, 386-389.

Krasnosel'skii, M. A. and Krein, S. G. (1952). An iteration process with minimal residuals [in Russian], Matematicheskii Sbornik NS, 31, 315-334.

Medgyessy, P. (1972). On unimodality of discrete distributions, Periodica Mathematica Hungarica, 2, 245-257.

Popoviciu, T. (1935). Sur les équations algébriques ayant toutes leurs racines réelles [in French], Mathematica (Cluj), 9, 129-145.

Seaman, J. W., Young, D. M. and Turner, D. W. (1987). On the variance of certain bounded random variables, The Mathematical Scientist, 12, 109-116.

Sharma, R. and Bhandari, R. (2013). On variance upper bounds for unimodal distributions, Communications in Statistics - Theory and Methods, in press.

Sharma, R., Shandil, R. G., Devi, S. and Dutta, M. (2004). Some inequalities between moments of probability distributions, Journal of Inequalities in Pure and Applied Mathematics, 5, 1-9.

Shisha, O. and Mond, B. (1967). Bounds on Differences of Means, In inequalities: Proceedings of a Symposium held at Wright-Patterson Air force base, Ohio, August 19-27, 1965 (Oved Shisha, ed.), Academic Press, New York, 293-308. 